\title{
GASMoC method: a phenol-free technique to detect acid-fast bacilli
}

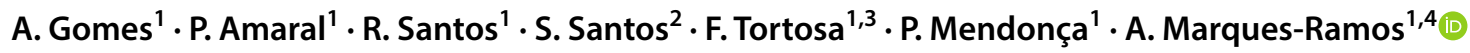

Accepted: 12 October 2019

(c) Springer-Verlag GmbH Germany, part of Springer Nature 2019

\begin{abstract}
The genre Mycobacterium includes a series of pathological species, such as M. tuberculosis, which is important for pathology laboratories to detect for a correct diagnosis. The Ziehl-Neelsen technique (ZNT) is the most commonly histochemical method used to detect these bacilli and uses a heated mixture of carbol-fuchsine, which contains basic fuchsine and phenol. Whereas the former component is responsible for the pinkish staining of acid-fast mycobacteria, the role of phenol is not completely understood and it has been suggested that its exclusion does not impact the detection ability of the ZNT. Since phenol is highly toxic and induces several injuries, the goal of this study is to determine the detection capacity of mycobacteria through a method that excludes the use of phenol. Accordingly, the GASMoC method, a modified ZNT that employs a solution of aqueous basic fuchsine heated at $37^{\circ} \mathrm{C}$, was tested on histological samples positive for mycobacteria and the results were compared to that of the ZNT. Data demonstrated that the GASMoC method was able to detect acid-fast bacilli (AFB) in all analyzed cases. Remarkably, microscopic inspection of mycobacteria was easier when the GASMoC method was applied. In conclusion, our study demonstrates that AFB detection does not require phenol and that the GASMoC method, a phenol-free technique, may substitute the ZNT in pathology laboratories.
\end{abstract}

Keywords Modified Ziehl-Neelsen technique $\cdot$ Mycobacteria detection $\cdot$ Safe acid-fast bacilli detection · Aqueous basic fuchsine $\cdot$ Carbol-fuchsine $\cdot$ Mycobacterium tuberculosis

\section{Introduction}

A. Gomes, P. Amaral, and R. Santos contributed equally to this work.

Electronic supplementary material The online version of this article (https://doi.org/10.1007/s00418-019-01825-0) contains supplementary material, which is available to authorized users.

A. Marques-Ramos

ana.ramos@estesl.ipl.pt

1 ESTeSL, Escola Superior de Tecnologia da Saúde de Lisboa, Instituto Politécnico de Lisboa, Av. D. João II, Lote 4.69.01, 1990-096 Lisbon, Portugal

2 Hospital de Vila Franca de Xira, R. Calouste Gulbenkian 1, 2600-009 Vila Franca de Xira, Portugal

3 Instituto de Anatomia Patológica, Faculdade de Medicina, Universidade de Lisboa, Av. Prof. Egas Moniz, 1649-028 Lisbon, Portugal

4 H\&TRC-Health and Technology Research Center, ESTeSL-Escola Superior de Tecnologia da Saúde, Instituto Politécnico de Lisboa, Lisbon, Portugal
Tuberculosis (TB) is an infection that is associated with the highest mortality rate worldwide, being considered by the World Health Organization (WHO) as one of the top 10 causes of death in 2016 (World Health Organization 2018a, b). This disease is caused by Mycobacterium tuberculosis, a bacilli that is transmitted by the inhalation of aerosolized particles that are either deposited in pulmonary alveoli, causing the so-called "pulmonary tuberculosis", or affect other parts of the body causing extra-pulmonary TB (Ghazaei 2018).

The mycobacterial cell envelope has a unique structure which features both: Gram-negative and Gram-positive bacteria, and is of key importance to its virulence and resistance to therapeutic agents (Vincent et al. 2018). The cell envelope is composed of the plasma membrane, a granular layer, the cell wall core (peptidoglycan and arabinogalactan layers), an outer membrane called "mycomembrane" and a loosely attached capsule (Angala et al. 2014). There is evidence that the mycomembrane has two leaflets: an external one, composed of glycolipids, phospholipids, acyltrehaloses, and 
phthiocerol dimycocerosates, and an internal one, consisting of mycolic acids (Angala et al. 2014; Vincent et al. 2018). These are long-chain fatty acids that constitute $40-60 \%$ of the cell envelope weight and are arranged in parallel conferring an extremely low fluidity to the cell wall (Angala et al. 2014; Vincent et al. 2018). The existence of mycolic acids makes the bacilli impermeable to lipophilic solutes (Liu et al. 1995). Thus, aqueous dyes commonly used in classic stains (such as in Gram stain) hardly penetrate the mycobacterial envelope (Liu et al. 1995). Additionally, mycobacteria are resistant to the removal of dyes with alcoholic and/or acidic solutions, thus causing these microorganisms to be designated as acid-fast bacteria or bacilli (AFB).

Several strategies have been developed to increase the permeability of mycobacteria to aqueous dyes to detect AFB in histological samples (Vasanthakumari et al. 1986). Initially, a heated $\left(40^{\circ} \mathrm{C}\right)$ alcoholic solution of methylene blue, potassium hydroxide and distilled water was used (Bishop and Neumann 1970). Subsequently, Bismarck brown V was introduced to stain the host cellular components and to remove the excess of AFB dye (Bishop and Neumann 1970). Since then, the used techniques have been based on a mordant, solution heating and AFB destaining (Bishop and Neumann 1970). In 1882, Erlich proposed to use an aniline basic fuchsine with a mineral acid as a destaining agent (Titford 2010). In the same year, Ziehl and Neelsen improved the method by replacing aniline with carbolic acid (phenol) and proposing a weak acid for destaining (Bishop and Neumann 1970). In 1887, Neelsen announced the Ziehl-Neelsen technique (ZNT), which is still one of the most widely used methods to diagnose AFB-caused diseases (Bishop and Neumann 1970), particularly in developing countries where TB is a leading cause of death (Ryu 2015).

The ZNT is a highly specific, rapid, simple and inexpensive method that stains AFB as a slightly curved and pink bacilli against a blue background (Fitzgerald et al. 2015). In the ZNT, the histological section is initially incubated with a heated carbol-fuchsine (CF) solution, then in a diluted acid solution (usually $1 \%$ hydrochloric acid in $70 \%$ ethanol) to remove the dye from the host tissue, which will then be stained by methylene blue (Ingen 2017). It has been suggested that both phenol and a high temperature are mandatory to increase the AFB permeability and, thus, allow the entry of basic fuchsine (Vasanthakumari et al. 1986). Since phenol is highly toxic, modifications of the original ZNT have been developed. One being the Kinyoun technique, which decreases phenol aerosol concentration since the phenol-fuchsine solution is not heated (Kinyoun 1915). Furthermore, it has been suggested that basic fuchsine alone allows satisfactory staining of AFB, thus avoiding the use of phenol (Della and Fail 2000; McCollough 2008). The goal of the present study was to determine the capacity for detection of mycobacteria by a method, called the GASMoC method, which eliminates the use of phenol and uses aqueous basic fuchsine at $37{ }^{\circ} \mathrm{C}$ to stain AFB. Our data demonstrates that $\mathrm{AFB}$ detection does not require phenol and that the GASMoC method may substitute the ZNT and the Kinyoun method in pathology laboratories.

\section{Materials and methods}

\section{Samples}

A total of 21 tissue specimens were used in this study: 13 cases were of human tissue previously diagnosed with TB, 7 of non-pathological human tissue (negative for the presence of AFB-negative control) and 1 of Zebrafish tissue positive for AFB (M. marinum - positive control). Since extra-pulmonary dissemination of $M$. tuberculosis has been reported in organs such as spleen, liver, intestine and lymph nodes (Peirse 2017), the aforementioned human specimens included 15 cases from extra-pulmonary tissue ( 9 previously diagnosed with TB and 6 non-pathological samples-negative control group) whereas the remaining cases were from lung tissue (4 diagnosed with TB and 1 from the negative control). The distinction between different organs was not made since the organism responds to $M$. tuberculosis in any anatomic location with the formation of caseous granulomas, with abundant purulence and causing local inflammatory destruction that hinders microscopic identification of the tissue (Peirse 2017).

Two sections $(3 \mu \mathrm{m})$ were prepared from each of the 21 tissues, totaling 42 formalin-fixed paraffin-embedded (FFPE) histological sections. To ensure similar AFB quantity between the two sections from each case, the corresponding sections were obtained contiguously. Two groups composing of 21 slides each ( 20 human cases and the Zebrafish positive control) were stained by the ZNT and GASMoC method.

\section{Staining}

The two groups of slides were submitted, in parallel, to the ZNT and the GASMoC method, according to the following protocol:

1. Dewax and hydrate histological sections from FFPE samples.

2. Incubate for 15 min at $37{ }^{\circ} \mathrm{C}$ with $1 \%$ carbol-fuchsine solution (Merck, USA): $1 \mathrm{~g}$ of basic fuchsine, $10 \mathrm{~mL}$ of $100 \%$ ethanol, $5 \mathrm{~g}$ of phenol and $100 \mathrm{~mL}$ of distilled water-ZNT; or $1 \%$ aqueous basic fuchsine solution: $1 \mathrm{~g}$ basic fuchsine (Merck, USA) and $100 \mathrm{~mL}$ distilled water-GASMoC method.

3. Wash in running tap water. 
4. Differentiate in acid-alcohol ( $1 \%$ hydrochloric acid in $70 \%$ ethanol) until the tissue section becomes light pink, and the solution is colorless.

5. Wash in running tap water, three times for 2 min each.

6. Counterstain in 5\% methylene blue: $5 \mathrm{~g}$ methylene blue (Merck, USA), $100 \mathrm{~mL}$ distilled water, for $15 \mathrm{~s}$.

7. Dehydrate through graded alcohols, clear in xylene and apply a coverslip using a resinous mounting medium.

The differentiation step (step 4) was initially performed at the ZNT slide and the duration was determined by microscopic inspection (pink AFB without background). The same time was applied on the GASMoC slide so as to ensure uniformity in the duration of each case.

The two groups of slides included a specificity test, where both methods were performed in parallel in AFBnegative human histological sections. In this test, it was possible to understand that both techniques were specific for AFB as they did not stain any tissue component, contrary to what had been observed in the positive controls included in the test.

\section{Sample evaluation}

With the ZNT and the GASMoC method, AFB are stained pink to red and the host tissue components are colored blue. Mycobacteria are slightly curved bacilli of about $2-4 \mu \mathrm{m}$ in length and $0.2-0.5 \mu \mathrm{m}$ in width (Fitzgerald et al. 2015). All the sections were evaluated microscopically according to a proper evaluation grid that included the case number, tissue type (pulmonary or extra-pulmonary), technique used (ZNT or GASMoC) and notes. Evaluation was performed at $400 \times$ and $1000 \times$ magnification, as indicated in figure legends. Two pathologists evaluated all 26 sections microscopically ( 13 for $\mathrm{ZN}$ and 13 for the GASMoC method) from the TB-positive cases, classifying each section as "positive"-indicating presence of AFB — or "negative"-indicating absence of AFB in the entire tissue. This criterion is based on the most recent WHO recommendations for routine evaluation of samples submitted to ZNT (World Health Organization 2015) and on the studies from Della and Fail (2000) and McCollough (2008). In addition to the mentioned criterion, evaluators made observations regarding the intensity of AFB staining (by comparing the pair of slides belonging to the same case) and the quality of the contrast (whether or not it allows the evaluation of the case). The remaining 14 non-pathological slides and 2 positive control slides were observed by one of the researchers based on the same assessment criteria.

\section{Statistical analysis}

Data were interpreted using the IBM SPSS ${ }^{\circledR}$ software and the statistical results were obtained through the McNemar's Chi square test $(P$ value $=0.05)$.

\section{Ethical considerations}

The $\mathrm{TB}+$ samples used in this study were kindly provided by hospitals in the Lisbon and Setubal districts. One of these provided five anonymized and TB+ paraffin blocks, from two connecting sections (each with $3 \mu \mathrm{m}$ ). The remaining $\mathrm{TB}+$ cases were provided as anonymized histological sections, by two other hospitals. The present investigation was registered with the Ethics Committee at the "Escola Superior de Tecnologia da Saúde de Lisboa".

\section{Results and discussion}

To determine whether or not the GASMoC method (modified ZNT that uses $1 \%$ aqueous basic fuchsine heated at $37{ }^{\circ} \mathrm{C}$ for $15 \mathrm{~min}$ ), is able to detect mycobacteria similarly to that of the ZNT, a total of 13 TB-positive cases were submitted to both techniques and evaluated by two pathologists. Seven non-pathological cases (negative controls) were also used and subjected to the same experimental conditions (Supplementary Tables S1 and S2).

The result between the two evaluators (evaluator \#1 and \#2 from Supplementary Table S1) was initially determined and it was found that both agreed in $92.3 \%$ of the cases, since 12 out of 13 cases with previous diagnosis of TB obtained the same result (Supplementary Table S1). Case number 11 was classified as positive for AFB with the GASMoC method and "doubtful" with the ZNT by evaluator \#1 whereas evaluator \#2 classified it as positive with both techniques (Supplementary Table S1). This discrepancy led us to exclude case number 11 from subsequent analysis.

Of the 19 evaluated cases (12 TB+ and 7 TB-), 11 were positive for the presence of $M$. tuberculosis with the ZNT (57.9\%) (Table 1, Fig. 1a) as opposed to 12 positive cases with the GASMoC method, using FBA (63.2\%) (Table 1, Fig. 1a). From the TB+ samples, the GASMoC method showed bacilli in 12 (100\%), as opposed to ZNT which only identified 11 (92.3\%) (Table 1, Fig. 2). By analyzing the negative controls, it was possible to observe that all were negative with both the ZNT and the GASMoC method (Supplementary Table S2).

Together, these results demonstrate that the GASMoC method efficiently and specifically detects AFB.

For the $\mathrm{TB}+$ case that was considered negative for the presence of M. tuberculosis with the ZNT (Table 1 and Case \# 4 from Supplementary Table S1), one of the evaluators 
Table 1 The GASMoC method is able to detect Mycobacterium tuberculosis

\begin{tabular}{lccccc}
\hline & \multicolumn{2}{c}{ Ziehl-Neelsen technique } & & \multicolumn{2}{c}{ GASMoC method } \\
\cline { 2 - 3 } \cline { 5 - 6 } & Positive & Negative & & Positive & Negative \\
\hline TB+ & 11 & 1 & & 12 & 0 \\
TB- & 0 & 7 & & 7 \\
Total & 11 & 8 & & 12 & 7 \\
\hline
\end{tabular}

A total of 19 samples (12 TB+ and $7 \mathrm{~TB}-$ ) were submitted either to the standard Ziehl-Neelsen technique or the GASMoC method. The obtained results are presented as the absolute frequencies of histological samples evaluated as positive or negative for acid-fast bacilli (please see Supplementary Tables S1 and S2)

$T B$ tuberculosis, positive presence of acid-fast bacilli, negative absence of acid-fast bacilli

pointed out that the presence of a technical artifact (a wrinkle) made the evaluation difficult (Fig. 3). This observation may thus justify the disagreement between the positive result with the GASMoC method and the negative result with the ZNT (Table 1). This indicates that the GASMoC method has the same capacity as the ZNT to detect AFB. To confirm this, a comparison between the detection capacities of both techniques was performed by applying the McNemar's Chi square test. As anticipated, there was no statistically significant difference between the discordant results of the ZNT and the GASMoC method $(X 2=3.84 ; P>0.05$; McNemar's Chi square test). These results demonstrate that the GASMoC method detects AFB and has the same detection capacity of the ZNT. These data are in line with previous studies from Della and Fail (2000) and McCollough (2008) that point out that phenol might be suppressed from ZNT (Della and Fail 2000; McCollough 2008). Nevertheless, in these studies, a $0.5 \%$ basic fuchsine solution is used whereas our protocol involves the use of a more concentrated solution of 1\%. (Della and Fail 2000; McCollough 2008). Indeed, the authors of the ZNT proposed the use of CF at a concentration of $1 \%$ although, later, WHO recommended the use of this solution at $0.3 \%$ (WHO 1998). As some studies have shown that $1 \% \mathrm{CF}$ solution is more sensitive at detecting $\mathrm{AFB}$ in infected specimens (namely M. tuberculosis), in 1998, WHO released new guidelines for staining that indicated the use of $1 \%$ CF (WHO 1998; Selvakumar et al. 2002). Since $1 \%$ is the recommended carbol-fuchsine concentration for laboratory use, this was also the concentration used in this study.

Additionally, our data demonstrate that the GASMoC method detects $M$. marinum present in zebrafish samples. Similarly, McCollough (2008) showed that heated aqueous basic fuchsine stains $M$. avium and other acid-fast pathogens of finfish and marine invertebrates (McCollough 2008). Furthermore, Della and Fail (2000) demonstrated that it is possible to detect M. avium in human samples (Della and Fail 2000). Together, these data indicate that aqueous basic fuchsine is useful in demonstrating various types of mycobacteria.

The similar detection capacity of AFB by the GASMoC method and the ZNT indicates that phenol is not necessary for basic fuchsine to enter the bacteria cell wall as was believed until now. Indeed, the method initially mentioned by Koch does not use this compound but rather an alcoholic solution of methylene blue and $10 \%$ potassium hydroxide heated to $40{ }^{\circ} \mathrm{C}$ (Bishop and Neumann 1970). In 1946, a theory was proposed regarding the mechanism of the CF action, which stated that phenol turns the aqueous dye more lipophilic and, thus, allows it to be joined with lipids (Lamanna 1946). Additionally, it is believed that fuchsine, once within the cell wall, associates with mycolic acids and the resulting hydrophobicity prevents its removal from the differentiating solution (Barksdale and Kim 1977; Lutwick 1995). Considering that the interaction of fuchsine with mycolic acids occurs in the absence of phenol (Annam et al. 2009), and in line with our results, we propose that the increase in temperature is sufficient to induce the permeability of the mycobacterial wall by breaking the lipid portion a

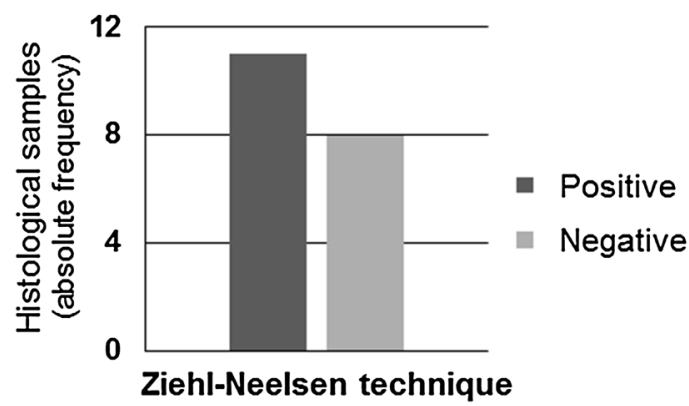

Fig. 1 M. tuberculosis detection by the standard Ziehl-Neelsen technique and the GASMoC method. Absolute frequency of the histological samples submitted either to the standard Ziehl-Neelsen technique b

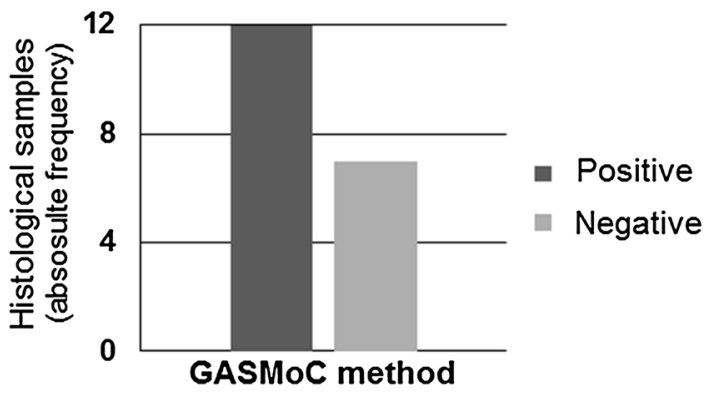

(a) or the GASMoC method (b) evaluated as positive or negative for mycobacteria. A total of 19 samples were considered-2 TB+ and 7 TB-. (Please see Supplementary Table S1 and S2) 


\section{Ziehl-Neelsen technique}
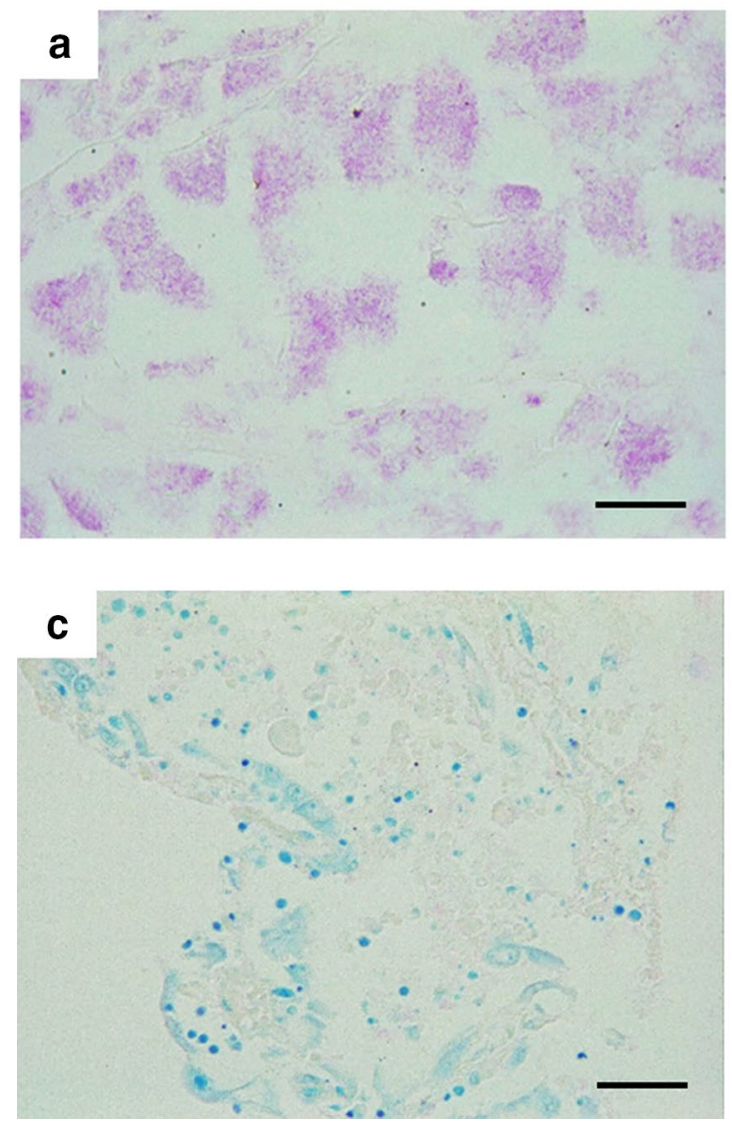

GASMoC Method
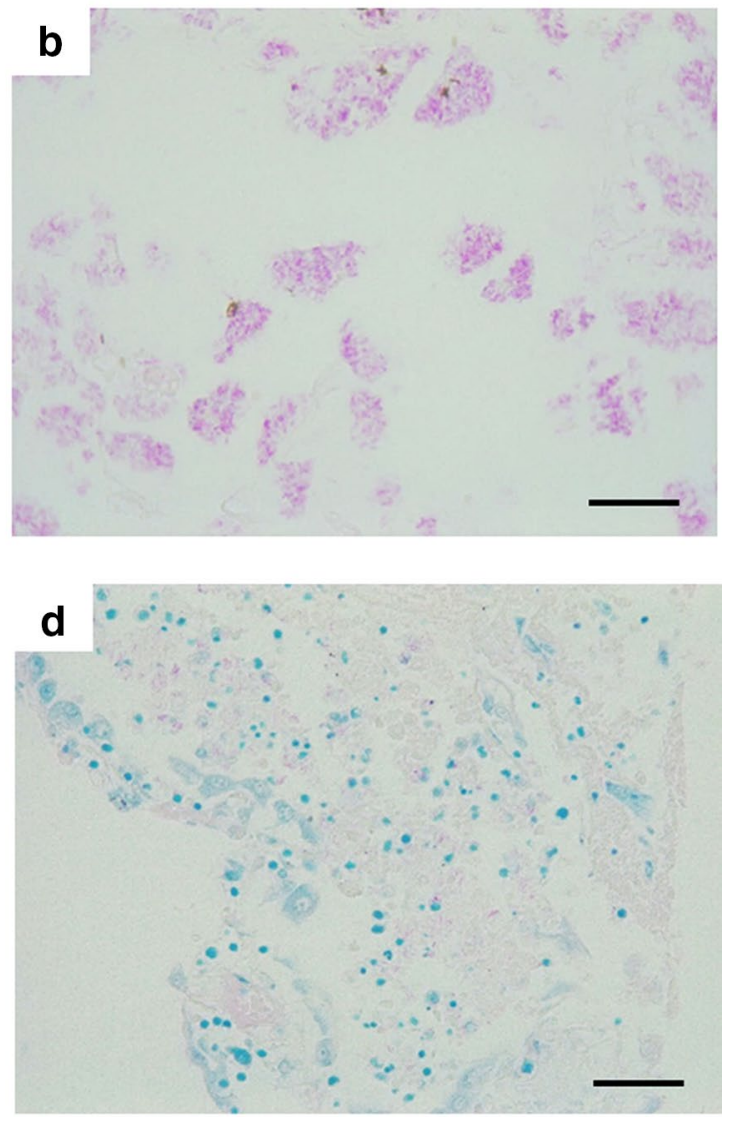

Fig. 2 Representative images of acid-fast bacilli stained with the conventional Ziehl-Neelsen technique (a, c) and the GASMoC method (b, d) in extra-pulmonary tissue samples with previous diagnosis of tuberculosis. Scale bar $=100 \mu \mathrm{m}(\mathbf{a}, \mathbf{b})=250 \mu \mathrm{m}(\mathbf{c}, \mathbf{d})$

\section{Ziehl-Neelsen technique}

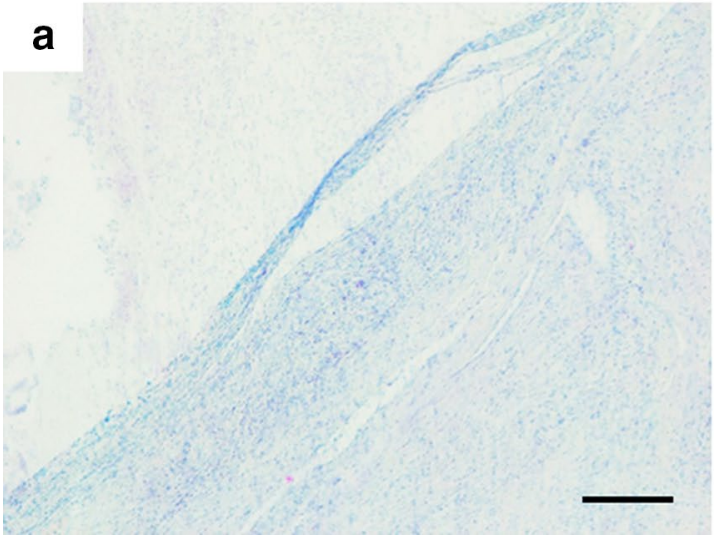

Fig. 3 Wrinkle present in a TB+ sample (case 4). Image of a TB+ sample (case 4 from Supplementary Table S2) that was evaluated as negative for the presence of $M$. tuberculosis using the standard Ziehl-

\section{GASMoC Method}

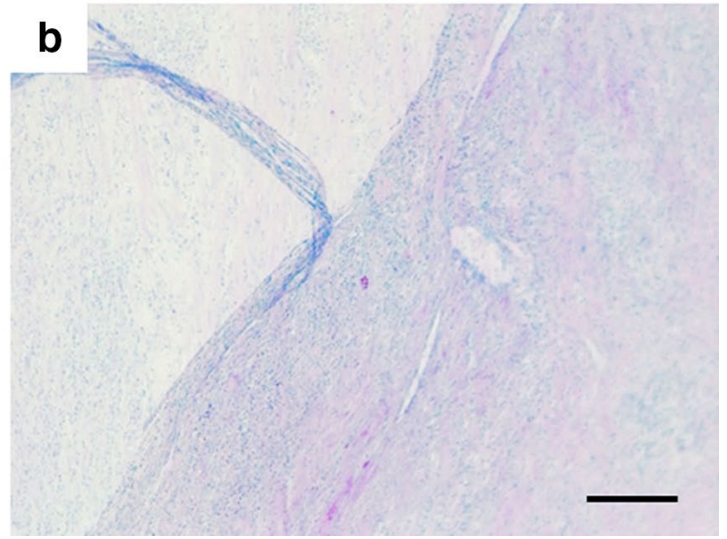

Neelsen technique (a) and positive using the GASMoC method (b). The image demonstrates a wrinkle that might obscure the presence of M. tuberculosis (a). Scale bar $=250 \mu \mathrm{m}$ 
and, thus, allowing the dye to enter. This suggestion supports the theory initially proposed by Lamanna (Lamanna 1946) that stated that phenol acts by making the dye liposoluble without interfering with the chemical reaction (Barksdale and Kim 1977; Vasanthakumari et al. 1986). Indeed, it has been shown that phenol does not induce lipid denaturation or causes chemical changes during the staining step (Donald and Lartigue 1962). Moreover, the substitution of phenol by lipophilic agents results in a satisfactory detection of bacilli (Ellis and Zabrowarny 1993; Clarke and McIntyre 1996), thus indicating that phenol is not mandatory for fuchsine staining.

Our data indicates that the GASMoC method enhances the AFB visualization compared to the conventional ZNT, as it provides a more intense pink color that makes it more noticeable against the blue background (Supplementary Table S1, Fig. 2a and b). As the incubation period of the dyeing and differentiating solutions were the same for each pair of slides submitted to GASMoC and ZNT, it is tempting to speculate that the observed difference may be a result of intrinsic characteristics of the dyeing solutions and their interactions with AFB. Whilst it is known that the basic fuchsine form develops with mycolic acids (Barksdale and Kim 1977; Lutwick 1995), it is our theory that the absence of the phenol, a weak acid, might free some fuchsine molecules with which it interacts and, thus, enables the dye to fully interact with mycolic acids. Further work must be performed to confirm this hypothesis.

Our study highlights the efficiency of the GASMoC method at detecting pathogenic mycobacteria in human samples thereby making a valuable contribution from a clinical point of view. The presented method omits the use of phenol and thus not only reduces the dangers associated with this highly toxic compound but also the costs associated with the AFB detection. It is, therefore, suggested that the GASMoC method replaces the Kinyoun or the ZNT in hospital practices (please see the supplemental material for protocol).

Acknowledgements We thank Prof. Anabela Graça, President of Escola Superior de Tecnologia da Saúde de Lisboa for allowing us to use the necessary reagents, consumables and equipment. We would also like to thank Gonçalo Borrecho, Sérgio Gomes and José Ferreira da Silva for providing the samples and to Prof. Carina Ladeira, for reviewing the article and providing useful comments.

\section{Compliance with ethical standards}

Conflict of interest The authors declare that there is no conflict of interest regarding the publication of this article.

\section{References}

Angala SK, Belardinelli JM, Huc-Claustre E, Wheat WH, Jackson M (2014) The cell envelope glycoconjugates of Mycobacterium tuberculosis. Crit Rev Biochem Mol Biol 49:361-399. https:// doi.org/10.3109/10409238.2014.925420

Annam V, Kulkarni M, Puranik R (2009) Comparison of the modified fluorescent method and conventional Ziehl-Neelsen method in the detection of acidfast bacilli in lymphnode aspirates. CytoJ 6:13. https://doi.org/10.4103/1742-6413.53887

Barksdale L, Kim KS (1977) Mycobacterium. Bacteriol Rev 41:217-372

Bishop PJ, Neumann G (1970) The history of the Ziehl-Neelsen stain. Tubercle 51:196-206

Clarke SC, McIntyre M (1996) Modified detergent Ziehl-Neelsen technique for the staining of Cyclospora cayetanensis. J Clin Pathol 49:511-512

Della Speranza V, Fail R (2000) Phenol myth unveiled! Were ZiehlNeelsen and Kinyoun mistaken? Histologic ${ }^{\circledR}$ Techn Bull Info Histotechn 33(2):21

Donald J, Lartigue GLF (1962) The chemistry of the acid-fast reaction. J Histochem Cytochem 10:611-618

Ellis RC, Zabrowarny LA (1993) Safer staining method for acid fast bacilli. J Clin Pathol 46:559-560

Fitzgerald DW, Sterling TR, Haas DW (2015) Mycobacterium tuberculosis. In: Bennett JE, Blaser MJ, Solin R (eds) Mandell, Douglas, and Bennett's principles and practice of infectious diseases. Saunders, New York, pp 2787-2818

Ghazaei C (2018) Mycobacterium tuberculosis and lipids: insights into molecular mechanisms from persistence to virulence. J Res Med Sci 23:63. https://doi.org/10.4103/jrms.JRMS_904_17

Ingen J (2017) Mycobacteria. Infectious Diseases, 4th edn. Elsevier, Amsterdam, pp 1645-1659

Kinyoun JJ (1915) A note on Uhlenhuths method for sputum examination, for tubercle bacilli. Am J Public Health 5:867-870

Lamanna C (1946) The nature of the acid-fast stain. J Bacteriol 52:99-103

Liu J, Rosenberg EY, Nikaido H (1995) Fluidity of the lipid domain of cell wall from Mycobacterium chelonae. Proc Natl Acad Sci USA 92:11254-11258

Lutwick LJ (1995) Tuberculosis. Springer Science, Dordrecht

McCollough C (2008) Application of an aqueous acid-fast staining technique to detect pathogens of aquatic species. Biotechnic Histochem 83:191-197. https://doi.org/10.1080/1052029080 2450780

Peirse M (2017) Extrapulmonary tuberculosis key points. Medicine. https://doi.org/10.1016/j.mpmed.2017.09.008

Ryu YJ (2015) Diagnosis of pulmonary tuberculosis: recent advances and diagnostic algorithms. Tuberculosis Respir Dis 78:64-71. https://doi.org/10.4046/trd.2015.78.2.64

Selvakumar N, Rahman F, Rajasekaran S, Narayanan PR, Frieden TR (2002) Inefficiency of $0.3 \%$ carbol fuchsine in ziehlneelsen staining for detecting acid-fast bacilli. J Clin Microbiol 40:3041-3043

Titford M (2010) Paul Ehrlich: histological staining, immunology, chemotherapy. Lab Med 41:497-498. https://doi.org/10.1309/ LMHJS86N5ICBIBWM

Vasanthakumari R, Jagannath K, Rajasekaran S (1986) A cold staining method for acid-fast bacilli. Bull WHO 64:741-743

Vincent AT, Nyongesa S, Morneau I, Reed MB, Tocheva EI, Veyrier FJ (2018) The mycobacterial cell envelope: a relict from the past or the result of recent evolution? Front Microbiol 9:2341. https ://doi.org/10.3389/fmicb.2018.02341

WHO (1998) Laboratory services in tuberculosis control. In: WHO (ed) Microscopy-Part II. WHO, Geneva, pp 27-30

World Health Organization (2015) Implementing tuberculosis diagnostics: a policy framework. WHO, Geneva

World Health Organization (2018a) The top 10 causes of death. http://www.who.int/news-room/fact-sheets/detail/the-top-10causes-of-death. Accessed 25 Nov 2018 
World Health Organization (2018b) Compendium of WHO guidelines and associated standards: ensuring optimum delivery of the cascade of care for patients with tuberculosis, 2 nd edn. WHO, Geneva
Publisher's Note Springer Nature remains neutral with regard to jurisdictional claims in published maps and institutional affiliations. 